\title{
Continuous nondestructive monitoring of larval feeding activity and development of the bamboo powderpost beetle Dinoderus minutus using acoustic emission
}

\author{
Hiroki Watanabe ${ }^{1} \cdot$ Yoshiyuki Yanase $^{1} \cdot$ Yoshihisa Fujii $^{1}$
}

Received: 3 August 2017 / Accepted: 5 November 2017 / Published online: 12 December 2017

(C) The Japan Wood Research Society 2017

\begin{abstract}
The life history and feeding biology of the bamboo powderpost beetle Dinoderus minutus remain poorly understood because the beetles' oviposition, development, and feeding take place inside bamboo culms. In this study, acoustic emission (AE) monitoring was applied for continuous nondestructive analysis of larval feeding activity and development from the first instar to adult eclosion. Newly hatched larvae were inoculated individually into pieces of madake (Phyllostachys bambusoides) culms. AE hits were recorded using single AE sensors fixed onto the bamboo pieces. Generation of AE hits indicated that larval feeding activity had begun after inoculation. Based on the time course of the hourly AE hit rate, the larvae were feeding constantly during each instar, and feeding activity only ceased during periods of ecdysis and pupation. Half of the individuals examined underwent seven instars and the other half underwent eight instars. The time course of AE hit rate per 5 min exhibited periodic cycles, where continuous meals were separated by inactive phases of ca. 5 min, with an average dominant period of each instar ranging between 0.76 and $2.19 \mathrm{~h}$. After correcting the AE data based on distance attenuation of $\mathrm{AE}$ waves, the tendency that $\mathrm{AE}$ amplitude increased as the larvae developed through ecdysis events became more apparent. AE monitoring continued after adult eclosion, and the feeding activity of the newly emerged adults continued almost ceaselessly during the Reifungsfrass period.
\end{abstract}

Keywords Powderpost beetle $\cdot$ Dinoderus minutus $\cdot$ Bamboo $\cdot$ Acoustic emission $\cdot$ Insect development

\section{Introduction}

Bamboo is an abundant natural resource in many regions, and bamboo culms can serve as a strong material with high machinability and aesthetic values. In Japan, bamboo culms have been used as construction and decorative materials in many traditional wooden houses, as well as for furniture and craft products. However, bamboo culms are susceptible to biodeterioration, even in seasoned conditions, and can be easily attacked by insects, thus limiting their usage.

Part of this paper was presented at the 66th Annual Meeting of the Japan Wood Research Society (Nagoya, Japan, 2016) and the XXV International Congress of Entomology (Orlando, Florida, USA, 2016).

\footnotetext{
Hiroki Watanabe

watanabe@h3news1.kais.kyoto-u.ac.jp

1 Graduate School of Agriculture, Kyoto University, Kitashirakawaoiwake-cho, Sakyo-ku, Kyoto 606-8502, Japan
}

The bamboo powderpost beetle Dinoderus minutus (Fabricius) (Coleoptera: Bostrichidae) is one of the most significant pests of felled bamboo culms in Japan. Both larvae and adults attack bamboo by boring tunnels inside the culms [1-3]. Infestation of D. minutus can begin by adult beetles entering into and ovipositing inside bamboo culms in the manufacturing process or during use. To prevent this, protection measures are necessary. When signs of infestation, such as entrance holes and piles of frass, are found, disinfestation measures are also needed. Techniques for early detection and accurate assessment of attack are desired when bamboo culms employed for construction or products are suspected of being infested. Establishment of these control measures requires detailed understanding of the biology of $D$. minutus, including information on their life history and feeding behavior.

Dinoderus minutus spend most of their life cycle in bamboo culms, where they feed, develop, and oviposit, thereby making analysis difficult. Therefore, studies on the biology of $D$. minutus have relied on the direct dissection of 
bamboo culms or tests using artificial diets [4-8], which may have affected the natural growth and behavior of the beetles. We consider that application of nondestructive testing techniques is a suitable approach to analyze the feeding and development of $D$. minutus inside bamboo culms.

We reported previously that the developmental stages of D. minutus were clearly distinguishable in images obtained by X-ray computed tomography (CT) and their bamboo consumption was assessed quantitatively [9]. We then traced the egg-to-adult development and tunneling process and quantified the growing body size and extending tunnel size in the entire larval period, with the aid of nutrient-containing filter paper for egg collecting [10]. On the other hand, because of the inability to conduct continuous monitoring, uncertainty remained regarding the activity of larvae between the CT scans. Therefore, we demonstrated directly that the chewing movements of the mandibles of D. minutus in feeding produced acoustic emission (AE) waves and proposed the effectiveness of AE monitoring for continuous analysis of their feeding activity [11]. Our preliminary experiment also suggested that periods of ecdysis and pupation could be estimated using $\mathrm{AE}$ [12]. Combined use of AE monitoring and $\mathrm{X}$-ray CT could comprehensively clarify the relations among the transition of feeding activity, larval development, and the amount of bamboo consumed by the larvae.

In this study, we nondestructively monitored the feeding activity of $D$. minutus inside bamboo pieces, continuously from the first instar to adult eclosion, and we determined the number of ecdysis events and the time period of each instar, and discussed the rhythmic patterns of feeding activity. X-ray CT scanning was used for two individuals to relate AE data to the developmental stages and bamboo consumption. Several additional experiments were conducted to directly observe the process of ecdysis, to estimate larval head capsule widths of all instars, and to discuss the effects of attenuation of $\mathrm{AE}$ waves on the $\mathrm{AE}$ monitoring of feeding activity. AE monitoring was continued after adult eclosion to analyze the pre-mating feeding activity of adult beetles.

\section{Materials and methods}

\section{Preparation of bamboo pieces and inoculation of larvae}

Bamboo pieces, 100 [longitudinal (L)] $\times 20$ [tangential (T) $\mathrm{mm}$, were prepared from air-dry internodes of madake (Phyllostachys bambusoides) culms, 6-7 mm [radial (R)] thick, felled in June 2014 in Kyoto Prefecture, Japan. Eggs of laboratory strains of D. minutus reared on madake culms were collected using laminates of nutrient-containing filter paper, as described previously [10], and newly hatched first instar larvae were collected randomly for inoculation into the bamboo pieces. A hole with a depth of $5 \mathrm{~mm}$ was drilled longitudinally on one end surface of each bamboo piece with a 2.5-mm drill bit and the hole was extended by $5 \mathrm{~mm}$ with a push pin. A newly hatched larva was placed individually into this hole, and the hole was closed using a round bamboo peg. Then, a piezoelectric AE sensor (R15 $\alpha$, Physical Acoustics Corp., USA) was glued onto the same end surface as where inoculation was performed with a cyanoacrylate adhesive (Fig. 1). After $2 \mathrm{~h}$, the pieces were placed vertically, with the inoculated end surface on top, in an environmental chamber and AE measurement was started. The environmental chamber was conditioned at $28{ }^{\circ} \mathrm{C}$ and $65 \%$ relative humidity, where the moisture content (MC) of the bamboo culms was $11 \%$. No light source was employed throughout the experiment. Also, because the sensors were insensitive to airborne noise, no soundproofing measures were employed.

To obtain ten replicates, 17 inoculated pieces were prepared. Of the 17 first instar larvae, 11 individuals successfully started feeding, but one individual ceased feeding after the first instar, suggesting that it had died in the process of ecdysis. The results and discussion are based on the surviving ten individuals.

Of these ten individuals, randomly chosen two individuals, AX1 and AX2, were also subjected to CT scanning using a microfocus X-ray CT system (SMX-160CTSV3S, Shimadzu Corp., Japan), usually with an interval of 3-5 days. Scans were performed daily when pupation and

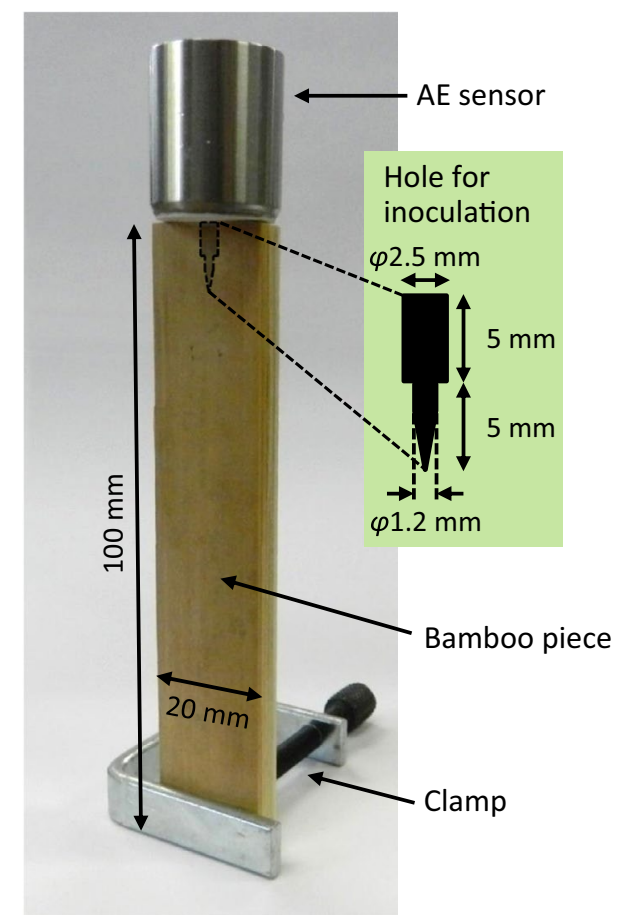

Fig. 1 Illustration of the bamboo piece with a first instar larva inoculated in the hole and an $\mathrm{AE}$ sensor glued onto the upper end surface 
adult eclosion approached. Methods of CT scanning and measurement of larval tunnel length and volume were based on our previous report [10]. The CT data of individuals AX1 and AX2 have been published in this report [10]. The eight individuals that were not used for CT scanning are referred to as $\mathrm{A} 1-\mathrm{A} 8$.

\section{AE measurement and analysis}

$\mathrm{AE}$ data were acquired using a 4-channel AE system (DiSP with AEwin, Physical Acoustics Corp., USA). Each of the AE sensors, with a resonant frequency of $150 \mathrm{kHz}$, was connected to the system via a preamplifier (1220A, Physical Acoustics Corp., USA), as outlined in Fig. 2. The AE signals detected by the sensor were filtered by a $100-400 \mathrm{kHz}$ bandpass filter and amplified by $40 \mathrm{~dB}$ in the preamplifier and by $20 \mathrm{~dB}$ in the system amplifier. The signals were then discriminated at a threshold of $80 \mathrm{~dB}$, and a burst signal that exceeded this threshold was counted as an AE hit. The time of detection and amplitude, i.e. the maximum (positive or negative) signal excursion during the hit, were recorded for each hit. The AE hits were determined using the hit definition time (HDT) of $100 \mu \mathrm{s}$, the hit lockout time (HLT) of $200 \mu \mathrm{s}$, and the peak definition time (PDT) of $40 \mu \mathrm{s}$. The amplitude values of $\mathrm{AE}$ hits were expressed in $\mathrm{dB}$ with a reference voltage of $1 \mu \mathrm{V}$.

We defined two parameters to express the AE data: hourly $\mathrm{AE}$ hit rate and mean maximum amplitude. Hourly AE hit rate was the number of $\mathrm{AE}$ hits detected in $1 \mathrm{~h}$, and mean maximum amplitude was calculated as the hourly mean of six maximum amplitude values recorded during 10-min intervals. In addition, frequency spectra of the time courses of the number of AE hits per 5 min calculated using fast Fourier transformation (FFT) were used to analyze the periodicity of larval feeding activity found in this experiment.
After adult eclosion, bamboo pieces containing adults were kept separately in glass bottles and AE measurement was continued. The threshold was raised to $85 \mathrm{~dB}$ prior to adult eclosion.

\section{Direct observation of ecdysis and pupation (additional experiment I)}

To verify that the larvae underwent ecdysis or pupated during periods of no AE detection, additional larvae were inoculated in bamboo pieces by placing inside holes made with a $1.5-\mathrm{mm}$ drill bit and the pieces were subjected to AE monitoring. When AE detection from these pieces stopped, the larvae were extracted and the process of ecdysis was observed using a microscope (VH-5000, Keyence Corp., Japan). A microscope image of the larva was recorded every $300 \mathrm{~s}$ in the environmental chamber. Two first instar larvae and five larvae in middle or later instars were used for observation.

\section{Measurement of distance attenuation of elastic waves in bamboo (additional experiment II)}

The amplitude attenuation of longitudinally propagating elastic waves per unit distance was measured by inputting continuous waves into bamboo culms of different lengths. Three bamboo specimens $[300(\mathrm{~L}) \times 20(\mathrm{~T}) \mathrm{mm}]$ were prepared from air-dry internodes of madake culms [6-7 mm (R) thick] at $8-9 \% \mathrm{MC}$ and with an air-dry density of $0.87-0.89 \mathrm{~g} / \mathrm{cm}^{3}$. Elastic waves were input from one end surface of each specimen, and the amplitude of the waves was measured at the other end surface. Then, the length of the specimen was shortened by $50 \mathrm{~mm}$ and the same procedure was repeated four times, until the specimen's remaining length was $100 \mathrm{~mm}$. The input signals of continuous sinusoidal waves with a frequency of $150 \mathrm{kHz}$ were generated using
Fig. 2 Schematic diagram of AE measurement system for bamboo pieces containing $D$. minutus larvae

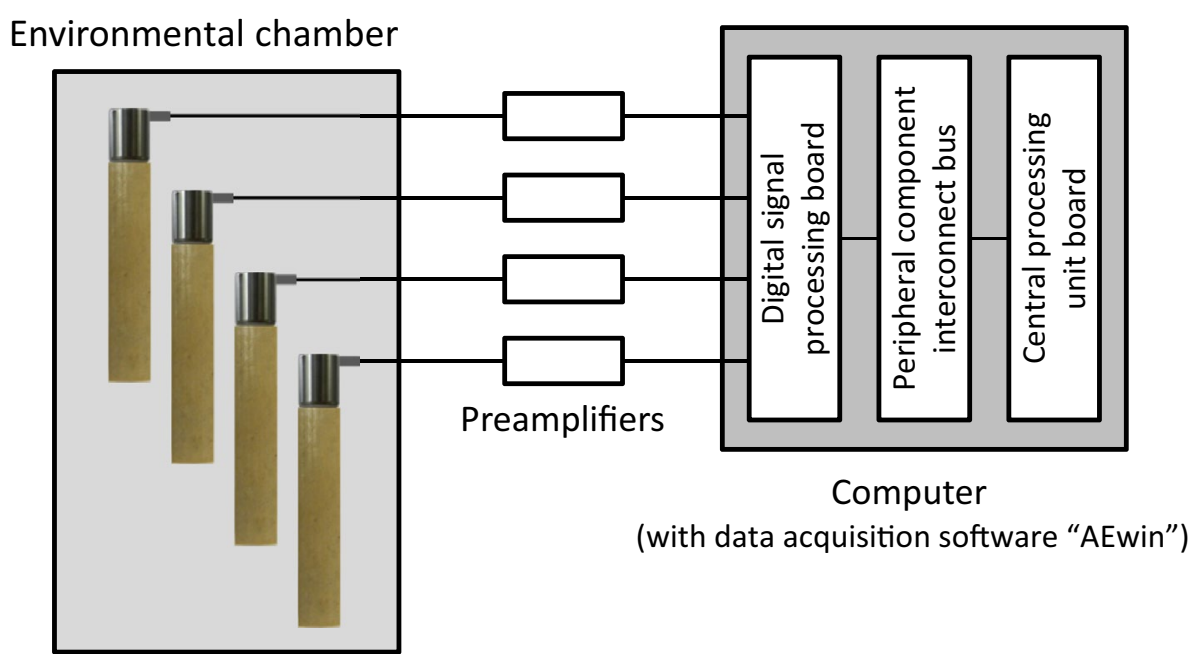


a digital function generator (DF1906, NF Corp., Japan) and were transduced and transmitted through a piezoelectric actuator (the same model as the AE sensors). To simulate AE waves generated by $D$. minutus larvae, a push pin was inserted into the end surface of the specimen, $2 \mathrm{~mm}$ below the inner surface, and the transmitter was fixed to the head of the push pin. A receiver AE sensor was attached to the other end surface of the specimen, and output signals detected by the receiver were acquired using an AE tester (AE9501A, NF Corp., Japan). A silicone grease was applied as an acoustic couplant between the transmitter and the push pin, and between the specimen and the receiver. The transmitter, the specimen, and the receiver were held together with a clamp. The amplitude of the output signals was displayed using a PC-oscilloscope (DSO-2090 USB, Qingdao Hantek Electronic Co., Ltd., China). The measurement system is summarized in Fig. 3. The attenuation in $\mathrm{dB} / \mathrm{mm}$ was calculated as the slope of the regression line relating the amplitude level of the output signals to the propagation distance (length of the specimen).

\section{Measurement of amplitude of AE generated by larvae of different instars (additional experiment III)}

To discuss the relationship of AE amplitude to larval head size and instars, AE signals generated by larvae of various body sizes were recorded, with the propagation distances kept constant. Four newly hatched first instar larvae and 13 larvae in middle or later instars were individually inoculated in bamboo specimens and the amplitude of AE hits were recorded. The head capsule widths of the first instar larvae were measured before AE measurement. The specimens $[15(\mathrm{~L}) \times 20(\mathrm{~T}) \mathrm{mm}$ ] were prepared from a single air-dry madake internode [6 $\mathrm{mm}(\mathrm{R})$ thick]. A longitudinal hole with a depth of $5 \mathrm{~mm}$ was made on one end surface of each specimen using a push pin or drill bits $(\varphi 1.2-1.4 \mathrm{~mm})$, depending on the larval body size, and a larva was placed in this hole. This resulted in a larva-to-sensor distance of $10 \mathrm{~mm}$. Each specimen was placed vertically on the face of

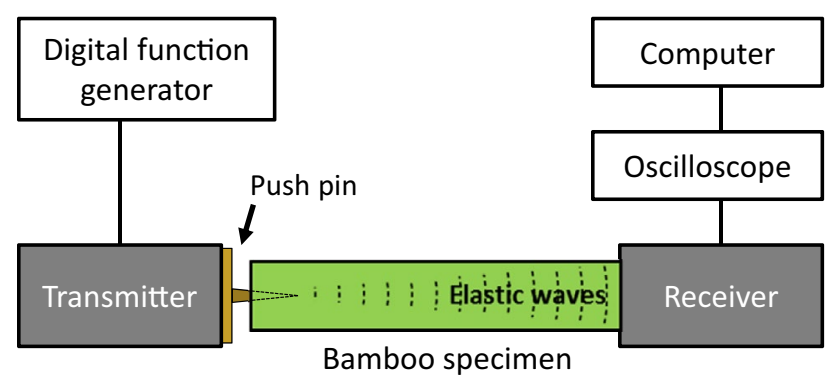

Fig. 3 Schematic diagram of the measurement system for attenuation of elastic waves in bamboo culms the AE sensor, with the inoculated end surface on top, with a silicone grease as an acoustic couplant. AE measurement was conducted using the AE system (DiSP with AEwin) in the environmental chamber for at least $2 \mathrm{~h}$ after the larva started feeding. The mean value of 12 maximum amplitude values recorded in 10-min intervals during a 2 -h period was calculated for each larva. This value is also referred to as mean maximum amplitude. After AE measurement, each of the middle or later instar larvae was extracted and killed in $99.5 \%$ ethanol. Because the head capsules of $D$. minutus larvae were partially hidden in the thoraces, except in the first instar, the head capsules of these extracted larvae were extruded from the thoraces to measure the widest part of the capsules.

\section{Estimation of head capsule widths of all instars (additional experiment IV)}

Dyar, who stated that the head capsule width of lepidopterous larvae follow a regular geometrical progression as they molt, recommended that the head capsule width of each instar be given in descriptions of larval stages [13]. However, the larvae monitored inside the bamboo pieces could not be used to measure the head capsule width. Because the validity of Dyar's rule has been confirmed in various insect species including coleopteran species, such as the mountain pine beetle Dendroctonus ponderosae [14] and curculionid weevils Sitona discoideus [15] and Pissodes castaneus [16], we applied his rule to $D$. minutus. The head capsule widths of 20 newly hatched first instar larvae and 20 final instar larvae in the prepupal stage were measured, and the growth ratio and the head capsule width of each instar were calculated. As shown below, the number of instars varied between seven and eight. Because the instar types of the prepupae were unknown, we estimated the head capsule width of each instar for both cases where the prepupae were all 7-instar type and 8-instar type.

\section{Results and discussion}

\section{Feeding activity and development from the first instar to adult eclosion}

Continuous generation of $\mathrm{AE}$ started within ca. 1 day after hatching. The AE hits detected from the bamboo pieces were attributable to larval feeding activity, based on our previous study [11], and even the activity of the smallest instar was detected. Considering the possibility of recording noise signals, we defined that the larvae were "active" when the hourly AE hit rate was 10 or higher, and otherwise they were regarded as being "inactive". Figure 4 shows the time courses of the hourly AE hit rate and mean maximum 

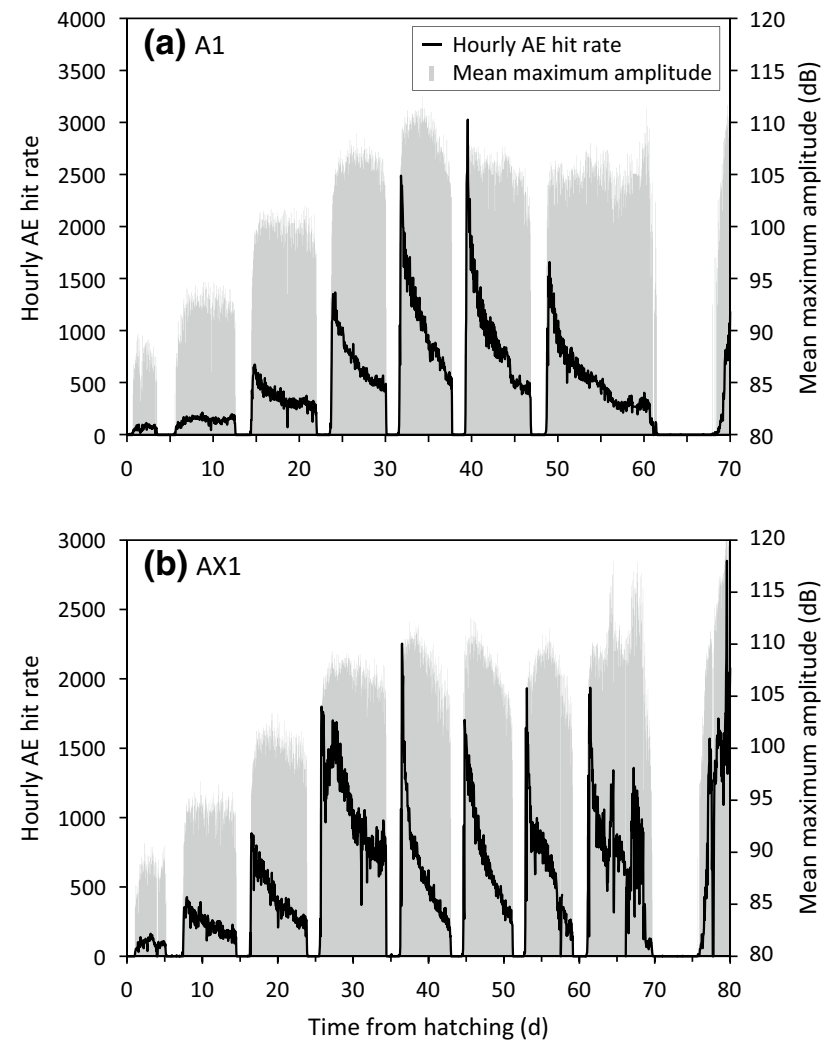

Fig. 4 Time courses of hourly AE hit rate and mean maximum amplitude of individuals A1 and AX1

amplitude of two individuals, A1 and AX1, as typical examples. They started continuous feeding at 0.6 and 1.0 days, respectively. However, feeding activity ceased between 3.5 and 5.5 days in individual A1 and between 5.2 and 7.3 days in individual AX1, and after that, periods of continuous feeding activity and periods of inactivity alternated with each other.

Direct observation (additional experiment I) confirmed that these periods of inactivity that lasted for ca. 2 days were due to ecdysis. Figure 5 shows the process of ecdysis of a middle instar larva. Three instances of ecdysis were directly confirmed for three different larvae in middle or later instars. These larvae underwent ecdysis 1.1 days after they became inactive on average. They did not consume their skins. Two other instances of ecdysis of two first instar larvae were also captured, although both larvae died after ecdysis, probably because of the disturbance. Based on the direct observation, it was revealed that an active period corresponded to an instar period and the number of inactive periods represented the number of ecdysis events.

After completing the seventh instar, individual A1 entered a longer inactive period lasting from 61.5 to 68.0 days (Fig. 4). Individual AX1 underwent an eighth instar before a long inactive period between 69.7 and 75.7 days. Daily CT scans of individuals AX1 and AX2 confirmed that these periods corresponded to prepupal and pupal stages. Individual AX1 in the final instar became inactive at 69.7 days, and CT scans confirmed that it turned into a prepupa between 69.9 and 71.0 days and pupated by 72.1 days. Direct observation of four final instar larvae showed that they pupated 1.5 days after they became inactive on average (additional experiment I). Individual AX1 emerged as an adult between 74.9 and 75.8 days. CT scans and AE measurement of individuals AX1 and AX2 suggested that adult beetles started feeding within ca. 1 day after eclosion. The feeding activity of adults is discussed in the final subsection.

The time courses of the hourly AE hit rate in Fig. 4 showed that the larvae were most active shortly after completing ecdysis, and the activity reduced as the next ecdysis or pupation approached, in a certain instar after the third instar. The mean maximum amplitude of AE hits was rather constant in a certain instar. When compared among instars, both the hourly AE hit rate and mean maximum amplitude tended to increase with the instar number, suggesting that larger amounts of strain energy were released by the chewing movements of the mouthparts as the larval head and mouthparts enlarged. However, after the fifth or sixth instar,
Fig. 5 Process of ecdysis of a larva. Exuvia is shown in circles. Image intervals are $300 \mathrm{~s}$
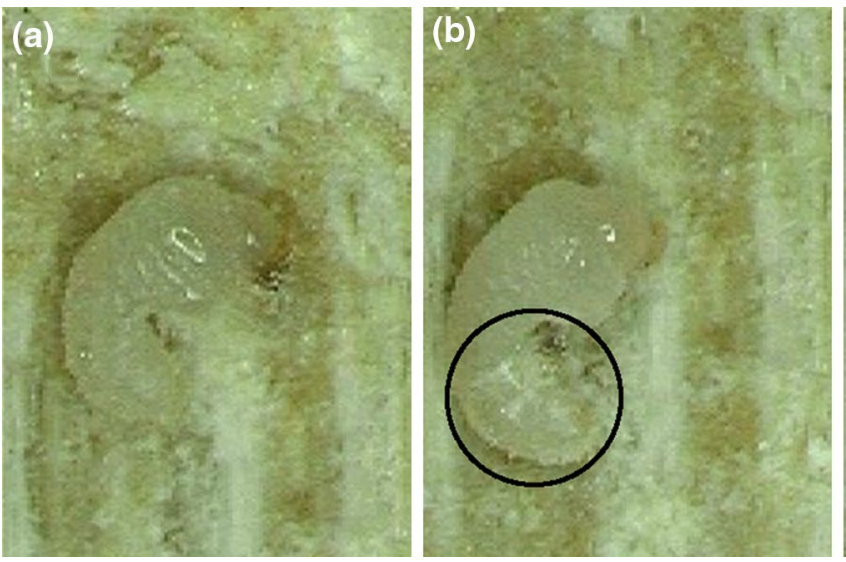
these tendencies became less clear. These patterns of the time courses of the hourly $\mathrm{AE}$ hit rate and mean maximum amplitude were observed in all ten larvae. Possible reasons for these patterns were that the larvae were actually less active in later instars and/or that attenuation of $\mathrm{AE}$ waves became significant as the larvae moved away from the sensors. This matter is discussed in the next subsection with reference to the attenuation characteristics of the AE waves.

Figure 6 shows the progression of instars of all ten larvae, as well as the durations of instars, inactivity due to ecdysis, and prepupal and pupal stages. Five larvae underwent seven instars ("7-instar type") and the other five underwent eight instars ("8-instar type"), suggesting that the number of instars can vary in this species. Although other numbers of instars were not found in this study because of the limitation of the number of $\mathrm{AE}$ measurement channels, it is possible that other instar types were present, with low abundance ratios. In related species, for example, variation in the number of larval instars was reported for the brown powderpost beetle Lyctus brunneus (Coleoptera: Bostrichidae) [17] and the tobacco beetle Lasioderma serricorne (Coleoptera: Anobiidae) [18]. The larval duration of 8-instar larvae was significantly longer than that of 7-instar larvae (Student's $t$ test, $p<0.01$ ). The adult body length was not significantly different between the instar types (Student's $t$ test, $p>0.05$ ), although the sample sizes may be too small to discuss differences among instar types. Sexual differences may be one of the factors causing the difference in the total number of instars. However, the sexes of the employed individuals were not identified in this experiment, limiting further discussion;

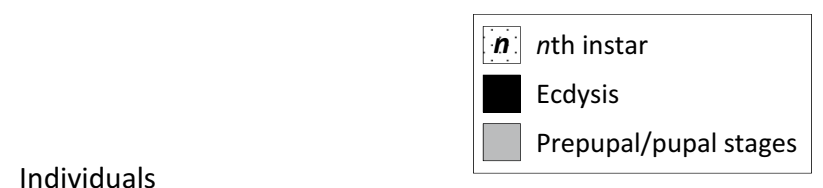

Individuals

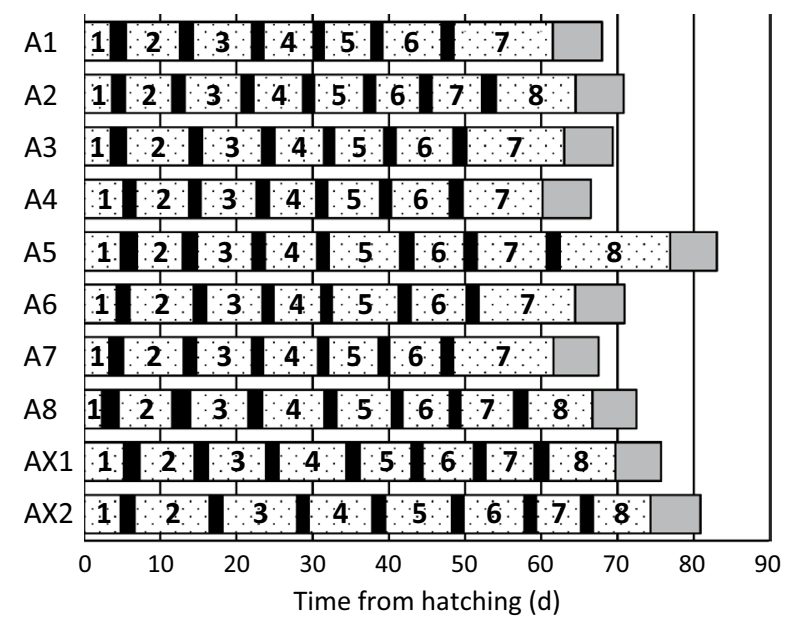

Fig. 6 Durations of larval instars, ecdysis, and prepupal/pupal stages the clarification of sexual differences in larval development is a subject for future analysis.

The number of instars revealed in this study does not agree with previous reports on D. minutus. Plank [4] estimated that $D$. minutus had four instars based on mandible lengths and Dyar's rule. Abood and Norhisham [6] found five instars based on head capsule widths and inspection of exuviae, by rearing larvae individually using cassava flour. Garcia and Morrell [5] reported that D. minutus larvae underwent four instars, but with no clear references to the methods of determining ecdysis. Some Japanese books [2, 3] mention that $D$. minutus have five larval instars, but without presenting evidence. The differences between our results and previous reports may be attributed to the accuracy of the conventional methods or to the variability of larval development among populations, regions, and rearing conditions. In addition, our results showed that first instar was the shortest and the final instars were the longest (Fig. 6), which was different from the report of Abood and Norhisham [6] who found that the final instar was the shortest. Although our results were in disagreement with the previous reports, we conclude that AE monitoring was an effective novel tool for nondestructively assessing ecdysis and instars.

The average larval duration and prepupal/pupal duration of the ten individuals were $66.3 \pm 5.7$ days (mean $\pm \mathrm{SD}$ ) and $6.2 \pm 0.3$ days, respectively. The average larval duration of the un-irradiated individuals, A1-A8, was $64.8 \pm 5.3$ days. This value was used in our previous report to show that there was no impact of irradiation on developmental period of the larvae examined using X-ray CT [10]. It should be noted that the larval durations measured from CT scanning and AE monitoring can be slightly different; in AE monitoring, the time from the end of feeding activity in the final instar to pupation, which was 1.5 days based on additional experiment I, was not included in the larval duration.

As Fig. 4 shows, again, the hourly AE hit rate was constantly above a certain level during each instar, suggesting that the larvae were feeding continuously throughout. However, time courses of the number of $\mathrm{AE}$ hits with a higher temporal resolution exhibited the rhythmic presence of short phases of inactivity alternating with feeding phases. In insect physiology, such feeding phases preceded and followed by a "gap" are called "meals" [19-21]. A typical example is shown in the time course of the number of AE hits per $5 \mathrm{~min}$ produced by individual $\mathrm{A} 1$ between 40 and 40.5 days in Fig. 7a, where meals lasting ca. $40 \mathrm{~min}$ interchange with gaps of ca. 5 min. Figure 7b shows the FFT spectrum of the time course of $\mathrm{AE}$ hits per $5 \mathrm{~min}$ in the sixth instar, i.e. between 39.2 and 46.9 days. In this spectrum, the highest peak is found at a frequency of 35.9 cycles/day, corresponding to a period of $0.67 \mathrm{~h}$. This means that the activity of the larva had a dominant periodicity with a period of $0.67 \mathrm{~h}$ during the sixth instar. The distribution of intensity around 

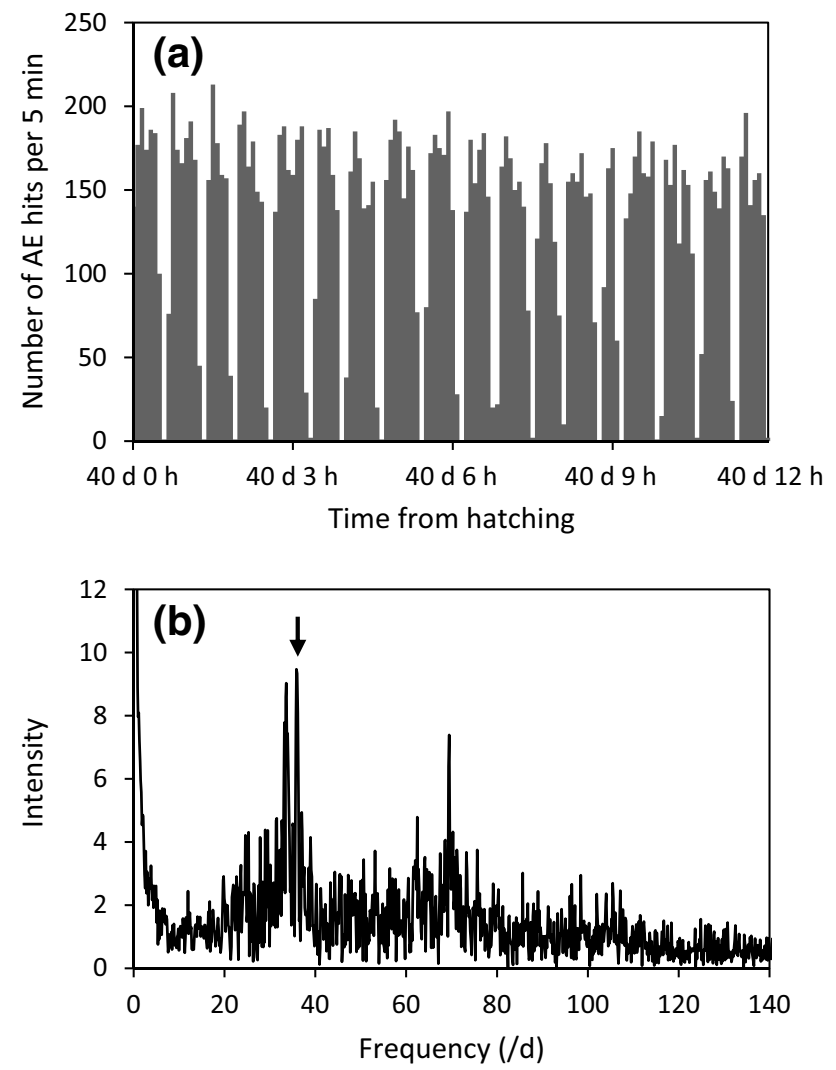

Fig. 7 Rhythmic pattern of feeding activity of individual A1. a Time course of the number of $\mathrm{AE}$ hits per 5 min between 40 and 40.5 days after hatching. b FFT spectrum of the time course of the number of $\mathrm{AE}$ hits per 5 min during the sixth instar, i.e. between 39.2 and 46.9 days. The highest peak, as indicated by an arrow, was found at the frequency of 35.9 cycles/day

the peak seemed skewed, with more components on the left (lower-frequency) side of the peak. The periods tended to lengthen as ecdysis approached and larval activity reduced, though this is not shown in Fig. 7a, and the shape of the distribution around the peak probably reflected this tendency. For simplification, the skewness is ignored in further discussion. Table 1 shows the average dominant period of periodic activity of each instar of all larvae. In the first and second instars, clear periodicity was not present except in the second instar of one individual. The periods of cycles tended to shorten as larval instars increased. The periods were much shorter than $24 \mathrm{~h}$, suggesting that the feeding activity was independent of circadian rhythms, and the variation of periods within and among instars and individuals under constant conditions suggested that the rhythm in feeding activity was endogenous. Feeding patterns with a certain periodicity independent of circadian rhythms have been reported for nymphs and larvae of several insects, such as the migratory locust Locusta migratoria [19], tobacco hornworm Manduca sexta [20], and silkworm Bombyx mori [21], and such patterns were estimated to be relevant to endogenous
Table 1 Dominant period of the rhythms of feeding activity in each larval instar, averaged over ten individuals $(n=10)$ except where noted

\begin{tabular}{ll}
\hline Instar & Period $($ mean \pm SD) $(\mathrm{h})$ \\
\hline 1 & ND \\
2 & $2.19(n=1)$ \\
3 & $2.04 \pm 1.16$ \\
4 & $1.15 \pm 0.31$ \\
5 & $0.97 \pm 0.32$ \\
6 & $0.76 \pm 0.13$ \\
7 (of 7-instar larvae) & $0.80 \pm 0.10(n=5)$ \\
7 (of 8-instar larvae) & $0.77 \pm 0.13(n=5)$ \\
8 & $0.86 \pm 0.11(n=5)$ \\
\hline
\end{tabular}

$S D$ standard deviation, $N D$ not detected

regulatory mechanisms [22]. Therefore, it is suggested that AE monitoring can also be applied to physiological analysis and analysis of rhythms, including circadian rhythms, in the activities of beetles.

In summary, AE monitoring was an effective tool for nondestructively detecting real-time feeding activity of $D$. minutus and determining the number of ecdysis events, the duration of instars, and larval and pupal developmental periods. Our work presents a novel, nondestructive yet reliable method for the analysis of feeding and clarifying life history, and this method should be applicable to many other woodboring insects. AE monitoring can be further applied to the evaluation of effects of environmental conditions on larval development and efficacy of disinfestation methods, such as heat or chilling treatments, for exterminating larvae inside bamboo culms. Real-time analysis using AE also indicated the presence of rhythms in the temporal transition of feeding activity within each instar period, which may reflect the endogenous regulatory mechanisms of the larvae. In addition, the results of this study showed the availability of $\mathrm{AE}$ monitoring for in situ detection of $D$. minutus, similarly to the cases of practical $\mathrm{AE}$ detection of termite attack in wooden structures [23-26]. The presence of rhythms in larval feeding activity may be useful for the differentiation of feeding activity from sporadic or stationary noise in in situ detection. Further experiments and discussion will be necessary for the optimization of in situ measurement apparatus.

\section{Effects of attenuation of AE waves}

In the previous subsection, we noted that the hourly AE hit rate and mean maximum amplitude did not increase with larval instars after the fifth or sixth instar (Fig. 4) and discussed that the reasons for these patterns may be that the larvae were actually less active in later instars and/or that attenuation of $\mathrm{AE}$ waves became significant as the larvae moved 
away from the sensors. To examine the extent of attenuation in our experimental conditions, we measured the distance attenuation of elastic waves propagating longitudinally in bamboo culms (additional experiment II) and corrected the AE data of individuals AX1 and AX2 using the attenuation value. Figure 8 shows the relationship between the propagation distance and amplitude level of $150-\mathrm{kHz}$ output signals for one bamboo specimen. The amplitude level decreased linearly with propagation distance, and the slope of the regression line was -0.12 . The average longitudinal attenuation of three specimens, at $8-9 \% \mathrm{MC}$, was $0.11 \mathrm{~dB} /$ $\mathrm{mm}$. Therefore, it was estimated that the amplitude of AE waves in bamboo culms decrease nearly by this value when using AE sensors with a resonant frequency of $150 \mathrm{kHz}$.

We then corrected the AE data of individuals AX1 and $\mathrm{AX} 2$ based on the distance attenuation measured in additional experiment II. We estimated the amplitude of each AE hit at the source by compensating for attenuation, using an attenuation value of $0.1 \mathrm{~dB} / \mathrm{mm}$ and the supposed propagation distance based on the linear interpolation of the distance between the larva and the sensor measured in X-ray CT images. The corrected AE hits were discriminated at a new threshold of $90 \mathrm{~dB}$. Figure 9 shows the time courses of corrected hourly $\mathrm{AE}$ hit rate and mean maximum amplitude of individual AX1. Both of these AE parameters increased with each instar, although the hourly AE hit rate at the beginning of the fifth instar was an exception for unknown reasons. Similar patterns were observed for individual AX2 as well. The correction based on attenuation indicated that the amplitude of $\mathrm{AE}$ waves, and thus the number of detectable signals (AE hits), always increased as the larvae underwent ecdysis events although this tendency became seemingly unclear after the fifth or sixth instar solely because of the attenuation of $\mathrm{AE}$ waves.

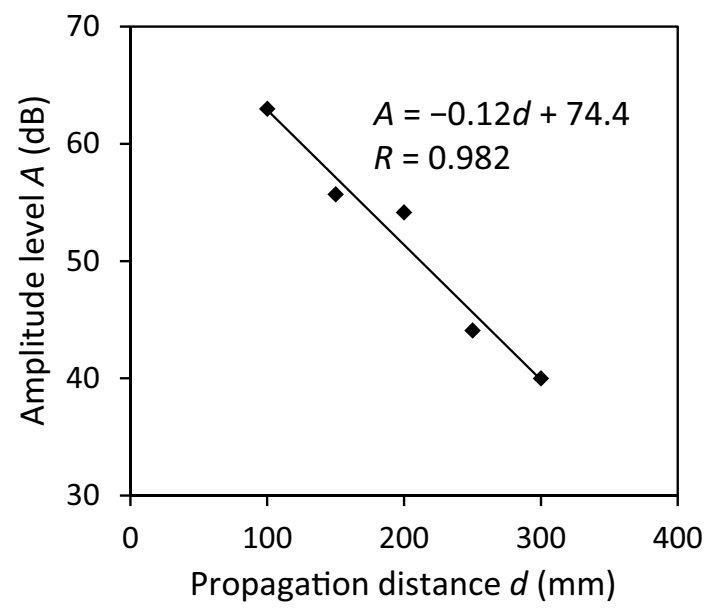

Fig. 8 Relationship between propagation distance and the amplitude level of output signals of $150-\mathrm{kHz}$ elastic waves

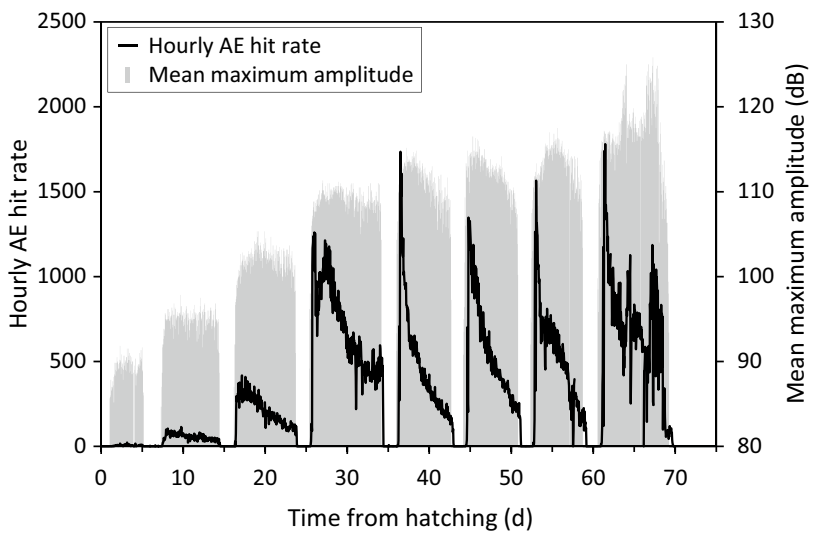

Fig. 9 Time courses of corrected hourly AE hit rate and mean maximum amplitude of individual AX1. The correction was based on the estimated attenuation of $\mathrm{AE}$ waves and the distance between the larva and the sensor

To further clarify the relationship between larval instars and produced AE amplitude, AE signals generated by larvae of different instars were measured with a fixed propagation distance of $10 \mathrm{~mm}$ in additional experiment III. Then, the head capsule widths of the larvae, except the four first instar larvae, were measured to estimate their instars. For instar estimation, a lookup table (Table 2) showing head capsule width of each instar calculated from 20 first instar larvae and 20 final instar larvae was made in additional experiment IV. This table lists the measured head capsule widths of the first and final instars, growth ratios calculated based on Dyar's rule [13], and calculated head capsule widths of intermediate instars for both 7- and 8 -instar types. Figure 10a shows the relationship between the head capsule width of larvae and mean maximum amplitude, the original result of additional experiment III.

Table 2 Estimated head capsule width of each larval instar

\begin{tabular}{|c|c|c|c|c|}
\hline \multirow[t]{2}{*}{ Instar } & \multicolumn{2}{|l|}{ 7-instar type } & \multicolumn{2}{|l|}{ 8-instar type } \\
\hline & $\begin{array}{l}\text { Head capsule } \\
\text { width }(\mathrm{mm})\end{array}$ & Growth ratio $r$ & $\begin{array}{l}\text { Head capsule } \\
\text { width }(\mathrm{mm})\end{array}$ & Growth ratio $r$ \\
\hline 1 & $0.119 *$ & $1.35 * *$ & $0.119 *$ & $1.29 * *$ \\
\hline 2 & 0.160 & & 0.153 & \\
\hline 3 & 0.215 & & 0.198 & \\
\hline 4 & 0.290 & & 0.255 & \\
\hline 5 & 0.390 & & 0.329 & \\
\hline 6 & 0.524 & & 0.424 & \\
\hline 7 & $0.706^{*}$ & & 0.547 & \\
\hline 8 & - & & $0.706^{*}$ & \\
\hline
\end{tabular}

*Mean of 20 measured values

**Calculated from measured head capsule widths of the first and final instars (*) based on Dyar's rule 
Fig. 10 Relationship between head capsule width of larvae and mean maximum amplitude (a) and relationship between larval instars and mean maximum amplitude on the assumption that the larvae were of 7-instar type (b) and 8-instar type (c). Dashed lines in $\mathbf{b}$ and $\mathbf{c}$ are the regression lines using values of all instars, and red solid lines are the regression lines using values between the fifth and final instars. Two asterisks (**) denotes that the correlation is significant at the 0.01 level

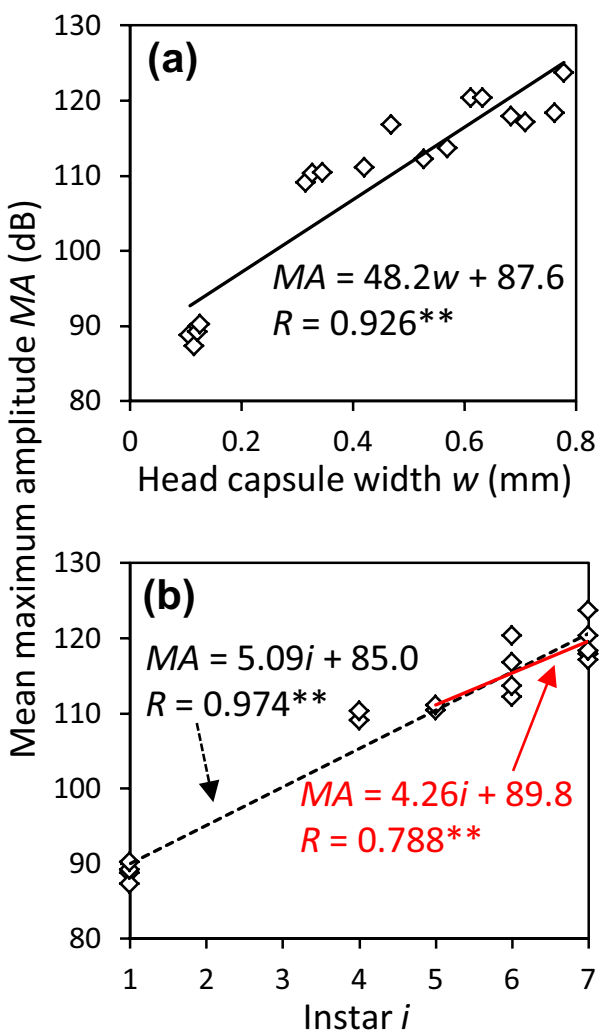

Figure 10b, c shows the relationship of mean maximum amplitude to larval instars, estimated based on Table 2 using the arithmetic means of head capsule widths of consecutive instars as borders. All of the correlations ( $R=0.926,0.974$, and 0.973 for Fig. 10a-c, respectively) were significant $(p<0.01)$, meaning that AE amplitude increased with larval head size and each instar. In addition, because the patterns in which maximum amplitude did not increase with each instar were only observed after the fifth or sixth instar, regression analysis was applied only to the mean maximum amplitude values of the fifth and later instars. The correlations $(R=0.788$ and 0.817 for Fig. 10b, $c$, respectively) were significant $(p<0.01)$ in both instar types. Therefore, it was confirmed that the amplitude of AE waves at their sources increased with head capsule width and the instar number.

For additional practical consideration, the distance attenuation measured in additional experiment II is useful in designing in situ detection systems for beetle attack in large bamboo culms in storage or in use. For example, the attenuation of $0.1 \mathrm{~dB} / \mathrm{mm}$ shows that $150-\mathrm{kHz}$ elastic waves propagate $0.5 \mathrm{~m}$ before attenuating by $50 \mathrm{~dB}$. Attenuation is estimated to be greatly influenced by the physical properties and conditions of the media, and the frequency of propagating waves, and the measurement system employed in additional experiment II can be applied to clarify the attenuation characteristics in detail.
Overall, it was shown that, when the larvae moved far away from the sensor, attenuation of $\mathrm{AE}$ waves became so significant that the larval activity could be underestimated. By compensating for attenuation, the correlation of $\mathrm{AE}$ amplitude with larval instars became clearer, reflecting the growth of the larvae. In the application of AE monitoring for continuous analysis of feeding by wood-boring insects, attenuation may not be problematic in separating instar periods from inactivity due to ecdysis or even in separating meals and subsequent (or preceding) gaps as long as the rearing media are of confined dimensions. However, when quantitatively comparing the level of feeding activity of different instars or individuals, it is desirable that the attenuation characteristics be evaluated in some manner, such as demonstrated in additional experiment II.

\section{Relationship between cumulative AE hits and bamboo consumption}

Previously, we clarified the relationship between the movements of the mandibles of $D$. minutus and $\mathrm{AE}$ generation [11]. However, the AE phenomenon is yet to be related to the actual amount of bamboo consumption by the larvae. In this subsection, we discuss the relationship between the number of AE hits and bamboo consumption using individuals AX1 and AX2. The tunnel volumes measured using X-ray CT images were used to represent bamboo consumption. 


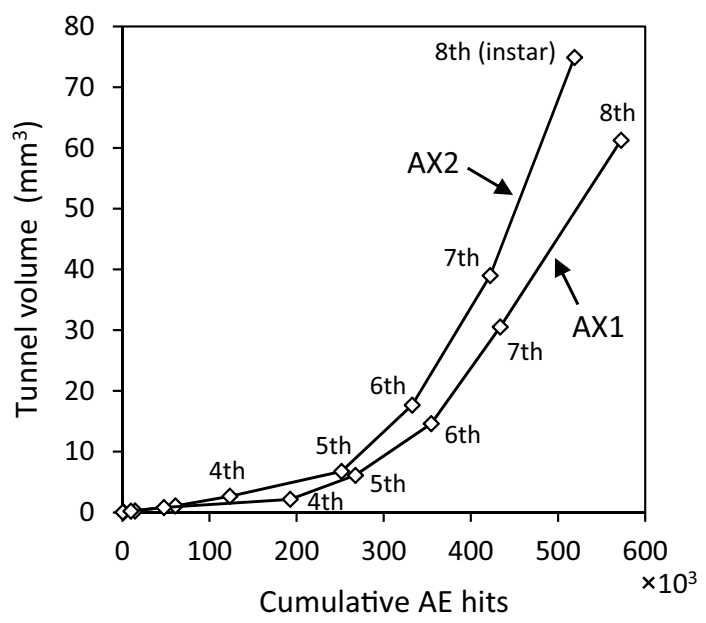

Fig. 11 Relationship between cumulative AE hits (corrected based on attenuation) and tunnel volume recorded from hatching until the end of each instar. Ordinal numbers represent instar numbers, excluding the first to third instars

The tunnel volume bored during each instar was estimated assuming that the tunnel volume increased linearly with time between two CT scanning dates and that the larvae did not extend tunnels during inactive periods of ecdysis. Figure 11 shows the relationship between cumulative AE hits (after correcting based on attenuation) and tunnel volume recorded from hatching until the end of each instar for the two larvae. The graphs in Fig. 11 confirm that the bamboo consumption increased as more AE hits were produced, although the relationship did not seem to be linear. A possible explanation for the rather concave shape of these curves was the growth of the mandibles; as the larvae enlarged through ecdysis events, larger amounts of bamboo were consumed by one bite of the mandibles. In addition, each of the two larvae consumed a total of $68.1 \mathrm{~mm}^{3}$ of bamboo on average, and the average percentage of bamboo consumption in each instar from first to eighth was $0.03,0.3,1.0,2.2,6.0,14,27$, and $49 \%$, respectively. This indicated that bamboo consumption increased greatly with each instar and suggests that detection and treatment in early larval instars would significantly reduce the damage caused by the larvae.

\section{Pre-mating adult feeding activity}

Analysis of pre-mating adult feeding (Reifungsfrass) was continued for ca. 10 days because our previous report showed that adults made exit holes in 8 days after adult eclosion on average [10]. Figure 12 shows the time courses of the hourly $\mathrm{AE}$ hit rate and mean maximum amplitude of individual A1, in continuation of Fig. 4a. It should be noted that a higher threshold value was employed for adult monitoring, so the adult hourly AE hit rate is not comparable with the

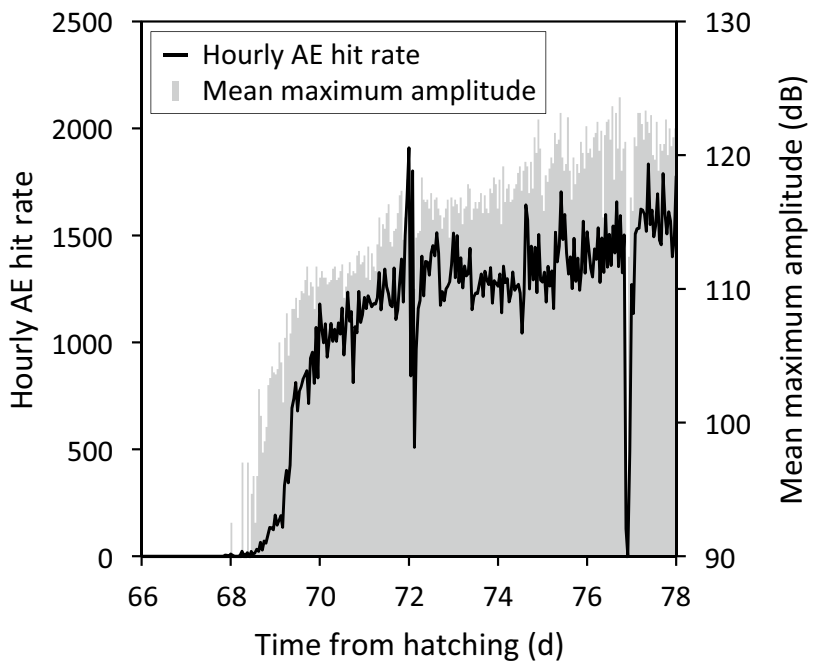

Fig. 12 Time courses of hourly $\mathrm{AE}$ hit rate and mean maximum amplitude after adult eclosion of individual A1. It should be noted that, because a higher threshold value was employed, the adult hourly AE hit rate is not comparable with the larval data shown in Fig. 4

larval data. As mentioned in the first subsection in "Results and discussion", adults were considered to have started feeding within ca. 1 day after eclosion. Figure 12 indicates that feeding activity continued almost ceaselessly during Reifungsfrass, although it was relatively low for 1-2 days after the adults started feeding. Adults produced AE waves of larger amplitude than the final instar larvae. Unlike larval feeding, there was no clear periodicity in the time courses of the number of AE hits per $5 \mathrm{~min}$. In future research, we plan to analyze feeding and tunneling activities during the ovipositional process, and the results will be compared to the feeding activity during Reifungsfrass.

\section{Conclusions}

In this study, AE monitoring was used to analyze larval feeding and development of $D$. minutus from the first instar. AE hits produced by feeding activity were detected, and the larvae showed almost constant feeding activity every hour during the instar periods but became inactive during periods of ecdysis and pupation. Therefore, AE monitoring proved to be an effective tool for measuring larval duration and the number of ecdysis events. The examined larvae underwent either seven or eight instars in total, which differed from previous reports on D. minutus. AE data also indicated the presence of periodicity in larval feeding; phases of short inactivity (ca. $5 \mathrm{~min}$ ) occurred at average intervals of $0.76-2.19 \mathrm{~h}$, which possibly reflected the physiology of the larvae. The AE amplitude increased with each larval instar, but because of the attenuation of $\mathrm{AE}$ waves, this relationship 
became unclear in later larval stages without additional corrections. Bamboo consumption increased as cumulative $\mathrm{AE}$ hits increased, and the non-linear relationship between these suggested that more bamboo was consumed by each bite as the larval instars progressed. The feeding activity of newly emerged adults continued almost ceaselessly, based on hourly AE hit rate, in the Reifungsfrass periods. These findings showed that we established a novel nondestructive method for the analysis of feeding and development of $D$. minutus and other wood-boring insects; however, AE attenuation in the media should always be taken into consideration. AE monitoring, together with $\mathrm{X}$-ray $\mathrm{CT}$ as discussed previously [10], will allow further investigation of feeding biology and life history, which should aid in the establishment of pest control measures.

Acknowledgements This work was supported in part by Grants-inAid for Scientific Research (Nos. 25242032, 26450229, and 17J04018) from the Japan Society for the Promotion of Science.

\section{References}

1. Wood Technological Association of Japan (1961) Mokuzai-hozon handbook (wood protection handbook) (in Japanese). Shokodo, Tokyo

2. Yamano K (1976) Kenchiku-konchu-ki (Insect pests of buildings) (in Japanese). Sagami Shobo, Tokyo

3. The Society of House and Household Pests Science, Japan (1995) Kaoku-gaichu-jiten (Encyclopedia of house and household pests) (in Japanese). Inoue Shoin, Tokyo

4. Plank HK (1948) Biology of the bamboo powder-post beetle in Puerto Rico. Bulletin no. 44. Federal Experiment Station in Puerto Rico, Mayaguez

5. Garcia CM, Morrell JJ (2009) Development of the powderpost beetle (Coleoptera: Bostrichidae) at constant temperatures. Environ Entomol 38:478-483

6. Abood F, Norhisham AR (2013) Larval development of the bamboo borer (Dinoderus minutus Fabricius) using individual rearing method. Pertan J Trop Agric Sci 36:55-66

7. Norhisham AR, Abood F, Rita M, Hakeem KR (2013) Effect of humidity on egg hatchability and reproductive biology of the bamboo borer (Dinoderus minutus Fabricius). SpringerPlus 2:9

8. Norhisham AR, Faizah A, Zaidon A (2015) Effects of moisture content on the bamboo borer Dinoderus minutus. J Trop For Sci 27:334-341

9. Watanabe H, Yanase Y, Fujii Y (2015) Evaluation of larval growth process and bamboo consumption of the bamboo powder-post beetle Dinoderus minutus using X-ray computed tomography. J Wood Sci 61:171-177

10. Watanabe H, Yanase Y, Fujii Y (2017) Nondestructive evaluation of egg-to-adult development and feeding behavior of the bamboo powderpost beetle Dinoderus minutus using X-ray computed tomography. J Wood Sci 63:506-513

11. Watanabe H, Yanase Y, Fujii Y (2016) Relationship between the movements of the mouthparts of the bamboo powder-post beetle Dinoderus minutus and the generation of acoustic emission. J Wood Sci 62:85-92

12. Watanabe H, Yanase Y, Fujii Y (2015) Evaluation of larval feeding activity of the bamboo powder-post beetle Dinoderus minutus using acoustic emission monitoring. International symposium on wood science and technology 2015 abstract book, Tokyo

13. Dyar HG (1890) The number of molts of lepidopterous larvae. Psyche 5:420-422

14. Logan JA, Bentz BJ, Vandygriff JC, Turner DL (1998) General program for determining instar distributions from headcapsule widths: example analysis of mountain pine beetle (Coleoptera: Scolytide) data. Environ Entomol 27:555-563

15. Frampton ER (1986) Determination of the number of larval instars of Sitona discoideus Gyllenhal (Coleoptera: Curculionidae) using probit analysis. N Z J Zool 13:107-111

16. Panzavolta T (2007) Instar determination for Pissodes castaneus (Coleoptera: Curculionidae) using head capsule widths and lengths. Environ Entomol 36:1054-1058

17. Iwata R, Nishimoto K (1985) Studies on the autecology of Lyctus brunneus (STEPHENS) (Coleoptera, Lyctidae): VI. Larval development and instars with special reference to an individual rearing method. Wood Res 71:32-45

18. Niiho C (1984) Ecological study of the tobacco beetle. Lasioderma serricorne (F.). II. Growth of tobacco beetles fed on bread crumbs (in Japanese). Jpn J Appl Entomol Zool 28:209-216

19. Simpson SJ (1982) Patterns in feeding: a behavioural analysis using Locusta migratoria nymphs. Physiol Entomol 7:325-336

20. Bernays EA, Woods HA (2000) Foraging in nature by larvae of Manduca sexta-influenced by an endogenous oscillation. J Insect Physiol 46:825-836

21. Nagata S, Nagasawa H (2006) Effects of diet-deprivation and physical stimulation on the feeding behaviour of the larvae of the silkworm, Bombyx mori. J Insect Physiol 52:807-815

22. Nagata S, Morooka N, Asaoka K, Nagasawa H (2011) Identification of a novel hemolymph peptide that modulates silkworm feeding motivation. J Biol Chem 286:7161-7170

23. Fujii Y, Yanase Y, Imamura Y, Okumura S, Oka S (1998) Detection of termite attack in wooden buildings with $\mathrm{AE}$ monitoring: case study at a traditional Japanese warehouse (in Japanese). Jpn J Environ Entomol Zool 9:101-105

24. Fujii Y, Yanase Y, Yoshimura T, Imamura Y, Okumura S, Kozaki M (1999) Detection of acoustic emission (AE) generated by termite attack in a wooden house. Proc IRG Annu Meet, Rosenheim, pp 99-20166

25. Yanase Y, Fujii Y, Okumura S, Imamura Y, Kozaki M (1999) Detection of termite attack in wooden buildings using AE monitoring: a case study at a house of wooden panel construction (in Japanese). Jpn J Environ Entomol Zool 10:160-168

26. Yanase Y, Fujii Y, Okumura S, Yoshimura T, Imamura Y (2001) Detection of termite attack of Incisitermes minor (Hagan) using AE monitoring (in Japanese). Jpn J Environ Entomol Zool 12:53-67 